\title{
Wetting Heterogeneities in Porous Media Control Flow Dissipation
}

\author{
Julie Murison, ${ }^{1, *}$ Benoît Semin,,${ }^{2,1}$ Jean-Christophe Baret, ${ }^{1}$ Stephan Herminghaus, ${ }^{1}$ \\ Matthias Schröter, ${ }^{1 \dagger}$ and Martin Brinkmann ${ }^{3,1}$ \\ ${ }^{1}$ Max Planck Institute for Dynamics and Self-Organization, 37077 Göttingen, Germany \\ ${ }^{2}$ Laboratoire de Physique Statistique, Ecole Normale Supérieure, UPMC Univ Paris 06, \\ Université Paris Diderot, CNRS, 24 rue Lhomond, 75005 Paris, France \\ ${ }^{3}$ Experimental Physics, Saarland University, 66123 Saarbrücken, Germany
}

(Received 27 June 2014; published 3 September 2014)

\begin{abstract}
Pressure-controlled displacement of an oil-water interface is studied in dense packings of functionalized glass beads with well-defined spatial wettability correlations. An enhanced dissipation is observed if the typical extension $\xi$ of the same-type wetting domains is smaller than the average bead diameter $d$. Three-dimensional imaging using $\mathrm{x}$-ray microtomography shows that the frequencies $n(s)$ of residual droplet volumes $s$ for different $\xi$ collapse onto the same curve. This indicates that the additional dissipation for small $\xi$ is due to contact line pinning rather than an increase of capillary break-up and coalescence events.
\end{abstract}

DOI: 10.1103/PhysRevApplied.2.034002

\section{INTRODUCTION}

Flow of immiscible fluids in porous solids is encountered both in nature and in many technological applications ranging from groundwater or oil reservoirs [1-3], soils, filtration membranes [4,5], fuel cells [6,7], or microfluidic systems [8-11]. In all of these instances, pore geometry and wall wettability are essential factors that control the dynamics of slow interfacial displacement [1,2]. Natural porous media such as rocks and soils typically exhibit a large variation in wettability linked to chemical heterogeneities of the rock and pore space $[12,13]$. A detailed understanding of the displacement processes down to the pore scale is important to optimize $\mathrm{CO}_{2}$ sequestration [14], secondary and tertiary oil recovery [2], and to design novel materials for filtering, energy conversion, and fuel cells [6,7]. Statistical descriptions which incorporate pore-scale geometry $[15,16]$ into macroscopic single-phase flow descriptions have been able to largely improve predictions of dissolution and chemical transport through porous media. The impact of heterogeneously wettable pore walls, however, has been studied, so far, only in highly complicated natural rock, restored samples [17], or oversimplified bead-pack models.

The simplest model of mixed wettability, a bead pack consisting of mixtures of monodisperse wetting and nonwetting spheres, has been well studied. In this case, it has been found that sample scale wettability can be described by averages of surface energies over several pores $[18,19]$. However, experimental and numerical studies of mixtures of wetting and nonwetting beads of different diameters indicate that the typical size of wetting domains should

\footnotetext{
*julie.murison@ds.mpg.de

matthias.schroeter@ds.mpg.de
}

play a significant role in fluid invasion processes [20-22]. These seemingly contradictory findings about the role of wetting heterogeneities on different length scales need to be resolved in order to formulate a predictive continuum theory of multiphase flows in porous media [2,23].

State-of-the-art studies using three-dimensional imaging of immiscible two-phase flows based on either x-ray microtomography $[24,25]$ or confocal microscopy [26] have, so far, focused on the role of geometric heterogeneity [2]. In these samples, percolation theory describes the droplet size distributions of the residual phase [25,27,28]. The model systems shown in Fig. 1 allow us to test the validity of these predictions in the presence of a defined wetting heterogeneity.

Our experiments clearly demonstrate that the typical extension and spatial correlation of differently wettable surfaces in a porous medium strongly influence the displacement of immiscible fluids. Random packings of monodisperse glass beads with suitably functionalized surfaces allow us to prepare model porous media with same-type

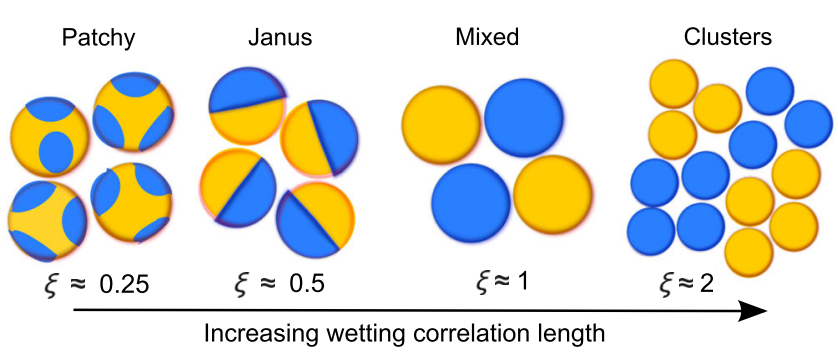

FIG. 1. Sketch of the four model porous media considered. Oiland water-wet surfaces are represented by orange and blue, respectively. All samples exhibit the same bead diameter $d$ and average surface energy but differ in the typical extension $\xi$ (normalized by $d$ ) of the water-wet domains. 
wettability domains of typical size $\xi$. We choose monodisperse bead packs in order to isolate effects caused by differences in wettability from those caused by local pore geometry. Capillary-pressure saturation (CPS) experiments are commonly used to characterize global sample wettability in core flood experiments and studies of synthetic porous media [3]. We combine these with x-ray microtomography, analyze the liquid-liquid distribution, and monitor the shape of the invading liquid front during the CPS experiments. Our experiments establish a link between CPS curves and pore-scale wetting heterogeneities.

\section{MATERIALS AND METHODS}

\section{A. Experimental setup}

CPS characteristics of each sample are measured in a self-built experimental setup. A sketch of the measurement apparatus is shown in Fig. 2. It consists of a cell containing the bead samples, a vertical translation stage which sets the height of the water reservoir to control the pressure difference between the two liquids, and a balance to measure the mass of oil displaced from the cell.

Samples of dense bead packs (mass approximately equal to $3 \mathrm{~g}$ ) are placed in a cylinder and held between two semipermeable membranes (top is hydrophobic, bottom is hydrophilic), each allowing only one liquid to pass through. We use composite membranes of two parts, a thick porous material imparting mechanical stability (both Porex, pore size $7-12 \mu \mathrm{m}$, height $1.5 \mathrm{~mm}$, XS-8259 is hydrophobic,

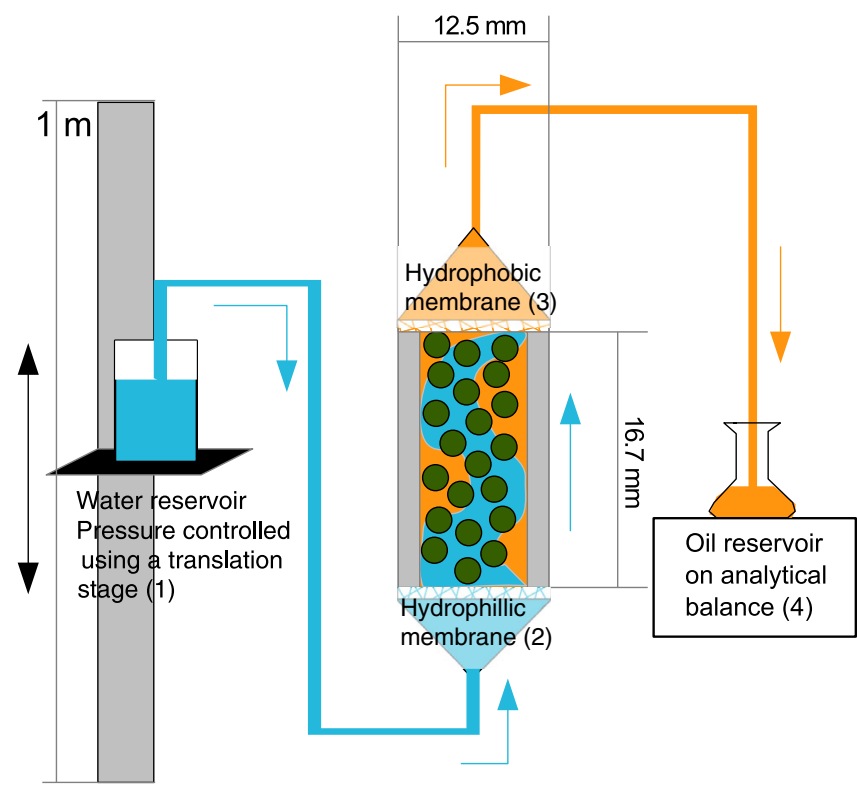

FIG. 2. Sketch of the experimental apparatus. The hydrostaticpressure difference $P_{\mathrm{c}}$ between water and oil is controlled by the height of the water reservoir (1). The hydrophobic membrane (3) is permeable for oil while the hydrophilic membrane (2) is permeable for water. The mass of the displaced oil is measured by an analytical balance.
XS-96190 is hydrophilic) and a thin membrane with very small pores preventing the flow of the nonwetting fluid even at high pressures (both Sartorius, pore size $0.45 \mu \mathrm{m}$, height $115-145 \mu \mathrm{m}$, PTFE is hydrophobic, nylon is hydrophilic). They are held together using small magnets (Supermagnete, $\varnothing=3 \mathrm{~mm}, h=1 \mathrm{~mm}$, nickel coating). The cell body is made of brass, with an inner Teflon tubing, of dimensions $\varnothing=12.5 \mathrm{~mm}$ and $h=16.7 \mathrm{~mm}$ with a total internal volume of $2.05 \mathrm{ml}$.

As immiscible liquids, we use hexadecane (Alfa Aesar, density $\rho=0.77 \mathrm{~g} \mathrm{~cm}^{-3}$ ), which is filtered through a 20-cm-high alumina column to remove surfactants, and millipore water as the aqueous phase. The interfacial tension (IFT) of the two liquids (hexadecane and millipore water) measured by pendant-drop tensiometry is $\gamma=$ $(51 \pm 1) \mathrm{mN} / \mathrm{m}$ (DataPhysics OCA 15EC goniometer). The CPS experimental apparatus is designed from materials which do not leach surfactants. This is checked by measuring the interfacial tension of millipore water against hexadecane in which each component of the cell is soaked and degassed for $24 \mathrm{~h}$. No significant changes in the IFT are observed.

Prior to running an experiment, it is necessary to remove all air from the cell. This is achieved by thoroughly degassing the membranes (in the wetting liquid) and the sample beads (in oil). Beads are pipetted into the cell while fully immersed in oil, avoiding entrapment of air bubbles. In the middle of each tubing, a three-way valve (Hamilton HV3) is placed, allowing the tubings to be flushed and primed with liquid preventing any trapping of air bubbles when the cell is connected. The top part of the cell and tubings [polyether ether ketone (PEEK), Upchurch Scientific, OD 1/16 in., ID 0.03 in.] are filled with oil, connecting the hydrophobic membrane to the oil reservoir, a glass Erlenmeyer flask placed on an analytical balance (Denver Scientific, 0.1-mg readout). To avoid force transmission to the balance, the tubing is fixed below the air-oil interface without touching the walls of the oil reservoir. Similarly, the bottom part of the cell and the hydrophilic membrane are connected to a $250-\mathrm{ml}$ glass bottle open to air through a PEEK tubing $(140 \mathrm{~cm})$.

The pressure difference $P_{c}=P_{w}-P_{o}$ between the continuous water $(w)$ and oil $(o)$ in the cell is set by the difference in hydrostatic pressures. The liquid-air interfaces of the water and oil reservoirs both have a large area (19.5 and $18 \mathrm{~cm}^{2}$, respectively) to ensure constant reservoir levels and negligible Laplace-pressure contributions. The hydrostatic pressure $P_{w}$ of the continuous water phase, and, consequently, the applied capillary pressure $P_{c}$, is controlled by vertically translating the water reservoir in steps of $10 \mathrm{~mm}$ (equivalent to $1 \mathrm{mbar}$ ) using a 1-m-high translation stage (Isel, Germany). In a full measurement cycle, the height of the water reservoir is gradually increased from -200 to $+200 \mathrm{~mm}$ and decreased back to $-200 \mathrm{~mm}$. During the entire experiment, the analytical balance 
measures the mass of oil displaced from the sample as a function of time. A custom-made LabviEw program is employed to read out the balance and to control the movement of the water reservoir.

The water reservoir is moved only when the mass of oil produced at the set height step reaches a steady value, i.e., when the standard deviation of the balance reading is less than $0.5 \mathrm{mg}$ during $200 \mathrm{~s}$. In order to assess the reliability of the stable water saturations measured at each height, we test other thresholds with longer counting times $(0.5 \mathrm{mg}$ during $400 \mathrm{~s}$ and $0.5 \mathrm{mg}$ during $600 \mathrm{~s}$ ). Prolonging the counting time does not result in any systematic variation. The height ramp of the water reservoir resulting from this protocol is one which changes rapidly when there is no or little change in the liquid saturations and slowly in the regions of large change, greatly improving the efficiency of the measurement without compromising on accuracy. The reduced time scales allow three to five full cycles in one to two days. Throughout the experiment, the temperature is regulated at $(21 \pm 0.5)^{\circ} \mathrm{C}$.

The reference height corresponding to zero capillary pressure is obtained by measuring the oil saturation change with the cell filled only with oil (no beads). At a certain reservoir height value, all the oil is displaced from the cell by the invading water. The reinvasion of oil during the following ramp-down occurs at the same height. Given the density of water and the acceleration of gravity, this calibration allows us to convert the height of the translation stage into an absolute capillary pressure.

From the known cell volume, the mass of beads loaded and their density $\left(\rho=2.51 \mathrm{~g} \mathrm{~cm}^{-3}\right)$, we calculate the liquid composition inside the sample. From the initial total mass of oil in the cell (ranging from 0.60 to $0.63 \mathrm{~g}$ ), and the mass of the produced oil, we compute the equivalent water saturation $S_{w}$. The water saturation is defined as the ratio of the water volume to the available interstitial volume of the sample in the cell. Using the experimental setup as described above and following the detailed protocol, we finally obtain capillary-pressure saturation curves by plotting the steady water saturation $S_{w}$ in the cell against the applied capillary pressure $P_{c}$.

\section{B. Image acquisition and processing}

Three-dimensional tomography data are collected using a GE nanotom with a tungsten target and a resolution of $12 \mu \mathrm{m}$ per voxel for the Janus, mixed, and cluster samples, and $6 \mu \mathrm{m}$ for the patchy samples (Fig. 5), and $17 \mu \mathrm{m}$ per voxel for the fluid morphology images (Figs. 8 and 10). Data are acquired using a current of $150 \mu \mathrm{A}$ and a voltage of $150 \mathrm{kV}$ to achieve the best contrast between all phases in the system. For each three-dimensional tomography image, 1440 two-dimensional images are collected with an exposure time of $250 \mathrm{~ms}$ using $2 \times 2$ binning to give a reconstructed file size of $1132 \times 1132 \times 1152$ pixels. The images are processed by Aviso Fire, a commercial software package designed for visualization and analysis of tomography data. To reduce noise, the reconstructed tomography data are filtered using a bilateral filter and a unsharp masking filter, both of which are edge preserving. Then, segmentation of the image is performed by grayvalue thresholding.

Experiments for the imaging of the fluid-fluid distributions are performed in a PEEK replica of the cell, allowing transmission of $\mathrm{x}$ rays through the sample without degradation of the cell or release of contaminant molecules into the aliphatic phase. A solution of $2 M \mathrm{KI}_{(\mathrm{aq})}$ as the aqueous phase is used to achieve $\mathrm{x}$-ray contrast between the phases and images of the liquid distributions are made with a resolution of $17 \mu \mathrm{m}$ per voxel.

\section{Sample design and characterization}

Different protocols to functionalize the glass beads and to pack them allow us to create model porous media with wetting heterogeneities of different spatial extensions. As shown in Fig. 1, the size of the same-type wettability domains $\xi$ ranges from smaller than a single pore containing several different wetting patches up to multiplebead diameters $d$. In all samples, we control the coverage such that $(50 \pm 5) \%$ of the surface is oil wet and $(50 \mp 5) \%$ water wet. The typical size $\xi$ of the surface domains is quantified by the correlation length of the wettability.

\section{Surface wettability}

All samples consist of packed spherical glass beads (MoSci) with average diameter $d=(375 \pm 25) \mu \mathrm{m}$ porosities in the range $0.38-0.40$ where the untreated surface of the glass beads constitutes the hydrophilic domains. Two different coatings that modify the local surface energy are employed to create the hydrophobic domains: gold and chlorotrimethoxysilane (CTMS). Gold beads are prepared by first sputtering the beads with gold (CreaVac, Dresden, thickness approximately equal to $200 \mathrm{~nm}$ ). The gold surfaces are then rendered hydrophobic by adding layers of hexadecane thiol (HDT). This is achieved by soaking gold-covered beads to a $1 \%$ volumetric HDT solution in hexadecane for $60 \mathrm{~min}$ at room temperature. Subsequently, the beads are rinsed in isopropanol and ethanol and dried in the oven at $75^{\circ} \mathrm{C}$ for $18 \mathrm{~h}$. The other coating CTMS consists of a chlorotrimethoxysilane (Sigma Aldrich) layer directly coated on the glass bead. CTMS surface coatings are prepared using a $0.5 \%$ volumetric solution of CTMS in toluene for $60 \mathrm{~min}$ at roomtemperature conditions, followed by curing in the oven at $75^{\circ} \mathrm{C}$ for $18 \mathrm{~h}$.

The wettability of both of these coatings is determined by measuring CPS curves on homogeneously coated beads (gold and CTMS) and compared with cleaned glass beads (Fig. 3). Both gold- and CTMS-coated bead packs exhibit hydrophobic behavior as determined by water-imbibition 


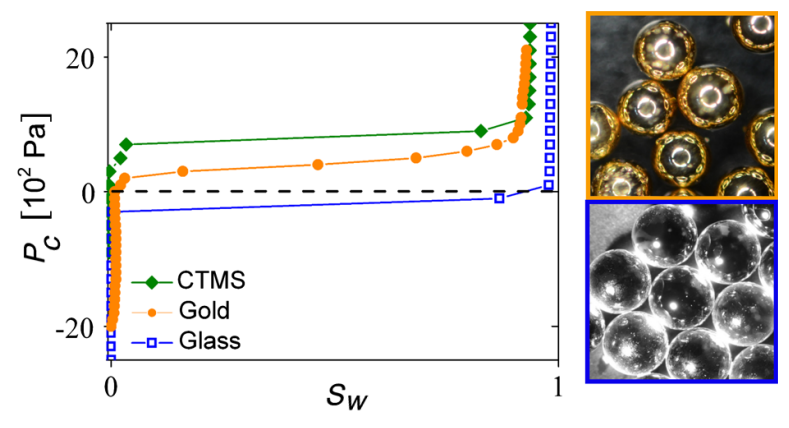

FIG. 3. Left: Second water-invasion curves for gold-coated, CTMS-coated, and cleaned glass beads. The untreated glass beads spontaneously imbibe the water into the packings (oil is displaced for capillary pressures $P_{c}<0$ ), whereas only forced imbibition (oil is displaced only for $P_{c}>0$ ) occurs in the case of the oil-wetting CTMS and gold-treated beads. Right: Pictures of gold-coated (top) and glass beads (bottom).

curves. In these hydrophobic samples, the water is able to invade the bead packing only at positive applied capillary pressures demonstrating the treated bead packs behave as oil-wet surfaces. In contrast, cleaned glass beads spontaneously imbibe water (imbibition at negative capillary pressure) and demonstrate the hydrophobic nature of the cleaned glass bead surfaces.

To ensure that there is no deterioration of the hydrophobic coatings or contamination of the surfaces during an experiment, we check that the hydrophilic beads remain hydrophilic and the hydrophobic remain hydrophobic. This is achieved by running a two-layer sample test with uncoated hydrophilic beads at the top and hydrophobic beads at the bottom. After the experiment, the beads are separated and their wettability checked by placing the beads in water. The hydrophobic beads form aggregates and minimize their contact with the water by trapping air bubbles between clusters of beads. By contrast, the hydrophilic beads do not aggregate or trap air between beads. This test shows that we do not have any spreading of the CTMS coating, unlike the results for octadecyltrichlorosilane (OTS) coatings in similar experiments [29].

\section{Spatial variation of wetting domains}

Variation of the spatial extension of the two wetting domains is achieved by applying different masking techniques in combination with the surface-modification protocols, gold, and CTMS. The sketches in Fig. 1 illustrate the four different sample types in our experiments. The four sample types and their respective masking protocols are as follows: (i) Patchy beads are created by masking the contact points in a granular pile with hexadecane and modifying only the exposed surface. This is achieved using a liquid hexadecane content of $2.0 \%$ with respect to the total sample volume. The sample is cooled to $-20^{\circ} \mathrm{C}$, solidifying the mask which prevents rearrangement of the bridges, and then the remaining exposed surface is the modified using
0.5 vol\% solution of CTMS in hexane. The amount of surface coverage can be controlled by the volume liquid content in the bridges and is approximately 50\% [30]. (ii) Janus beads with one hydrophobic and one hydrophilic hemisphere are created by sputtering only the top surface of the glass beads with gold. (iii) Mixed samples consist of a random mixture of equal weights of either gold- or CTMStreated beads and cleaned glass beads. The fully coated gold beads are created by tumbling the beads during the sputtering process. (iv) Cluster samples are made from equal-weight mixtures of homogeneously wet clusters of approximately 30 spheres. These clusters are made by pushing beads partially wetted with hexadecane through a 1-mm grating and then freezing the hexadecane, resulting in bead clusters of a defined size and homogeneous wettability. Bead clusters of both wettabilities are then mixed and packed together to create the cluster samples.

\section{Measurement of the correlation length}

The typical extension of the wetting domains $\xi$ is extracted from the probabilities $p_{i j}(r)$ to find a point on the surface of a bead and another point on the surface of the same or any other bead in the sample at distance $r$ to have wettabilities $i$ and $j$. Based on these statistical data, we can define $\xi$ of the wetting domains as a correlation length of wettability.

The spatial distribution of different wetting surfaces is obtained from x-ray tomography images of dry samples. To this end, we design special patchy and Janus samples with x-ray contrasting surface domains. For the mixed and cluster samples, basalt and glass beads are used to represent beads of different wettability. For the Janus sample analysis, we employ gold-sputtered beads with a thick layer of gold (approximately $1 \mu \mathrm{m}$ compared to approximately $200 \mathrm{~nm}$ for the samples used in the CPS experiments). For the patchy samples, fully-silver-coated beads are wetted with $2 \%$ of the sample volume of Norlands optical adhesive no. 89, which is cured under UV light. The exposed silver is then etched away from the surface using a 4:1:1 mixture of $\mathrm{MeOH}: \mathrm{NH}_{4} \mathrm{OH}: \mathrm{H}_{2} \mathrm{O}_{2}$. The remaining silver is quantified using $\mathrm{x}$-ray tomography. The tomography images are segmented using gray-value thresholding, such that each wetting surface of the beads corresponds to a different phase. Voxels belonging to the different surfaces are labeled blue and orange; see Fig. 5.

To compute the numerical value of the correlation length from the tomography data, we first determine the probabilities $p_{i j}(r)$ to find surface voxels of color $i$ and $j$ at a distance $r$. Figure 4 shows the probabilities for all four sample types. The indices $\alpha$ and $\beta$ stand for oil and water wet, respectively, and correspond to the colors orange and blue used in Figs. 1 and 5. As expected, the probabilities level off and become constant at large distances $r \gg d$ for all four sample types. This indicates the absence of longrange spatial correlations, which could potentially arise 


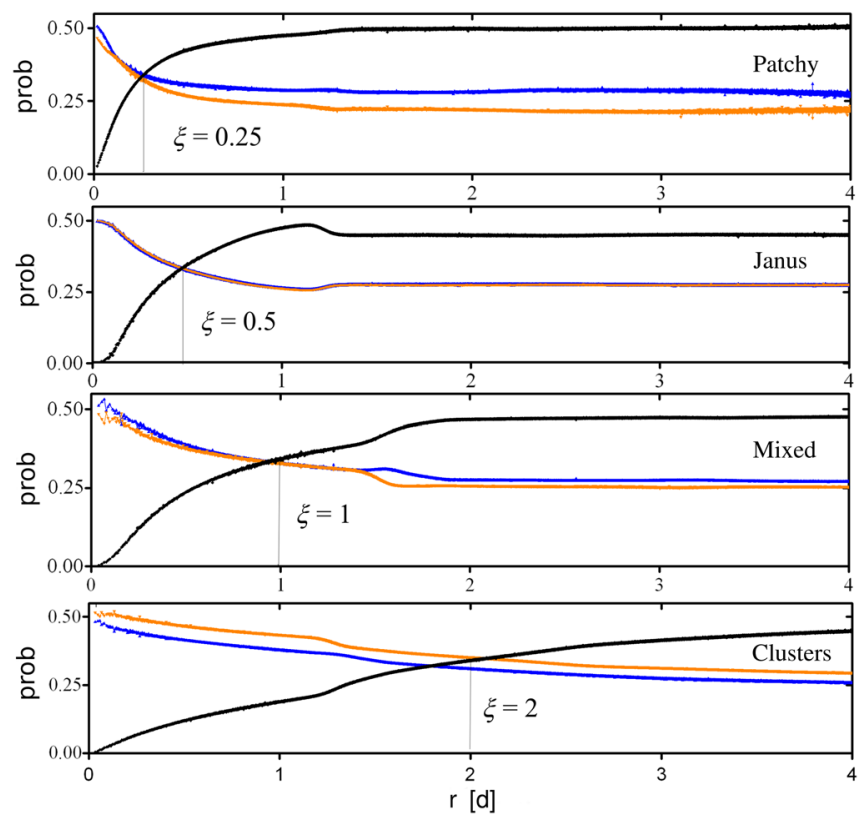

FIG. 4. Probabilities $p_{i j}$ of finding a pair of surface voxels with wettability $i$ and $j$ at a distance $r$ where the indicies $\alpha$ and $\beta$ stand for oil and water wet surfaces, respectively. $p_{\alpha \alpha}$ is shown in orange, $p_{\beta \beta}$ in blue, and $p_{\alpha \beta}$ in black. The distance $r^{*}$ at which the curves cross is indicated by the gray dashed line and determines $\xi$.

from orientational ordering of the beads. For all samples, we find plateaus $p_{\alpha \alpha} \approx 0.25, p_{\beta \beta} \approx 0.25$, and $p_{\alpha \beta} \approx 0.5$ for $r \gg d$, in agreement with the measurements of the areal fraction of different wettable surface domains.

The correlation length used to quantify the characteristic size $\xi$ of the surface domains is defined as the distance $r^{*}$ where the blue curves $p_{\alpha \alpha}(r)$ and the black curves $p_{\alpha \beta}(r)$ in Fig. 4 cross. The difference in $r^{*}$ between the crossing points of the blue and black curves, and the orange and black curves, is taken as the error for $\xi$. Our correlation length measurements yield values $\xi \approx 0.25 d$ for the patchy samples, $\xi \approx 0.5 d$ for the Janus samples, $\xi \approx d$ for the mixed sample, and $\xi \approx 2 d$ for the cluster samples; cf., also, Fig. 4. The values agree well with heuristic estimates of $\xi$ based on the geometry and spatial distribution of the modified surfaces.

\section{RESULTS AND DISCUSSION}

\section{A. Capillary-pressure saturation curves}

Following the protocol described the experimental section (Sec. II), capillary-pressure saturation curves are measured multiple times for the four different sample types. The liquid composition is described in terms of the water saturation, $S_{w}$, which is the volume of water inside the cell divided by the total interstitial volume of the sample. An example curve for each sample type is shown in Fig. 5. From the CPS curves, we extract the irreducible water and oil saturations $S_{w, i}$ and $S_{o, i}$, respectively, and the hysteresis loop opening, $\Delta P_{c}$.

\section{Irreducible water and oil saturations}

The irreducible saturation refers to the volume of liquid trapped inside the bead pack at the end of a water- or oilinvasion cycle. Because the trapped liquid bodies lost hydraulic connection to their respective reservoirs, they cannot be removed by any further increase (or decrease) of
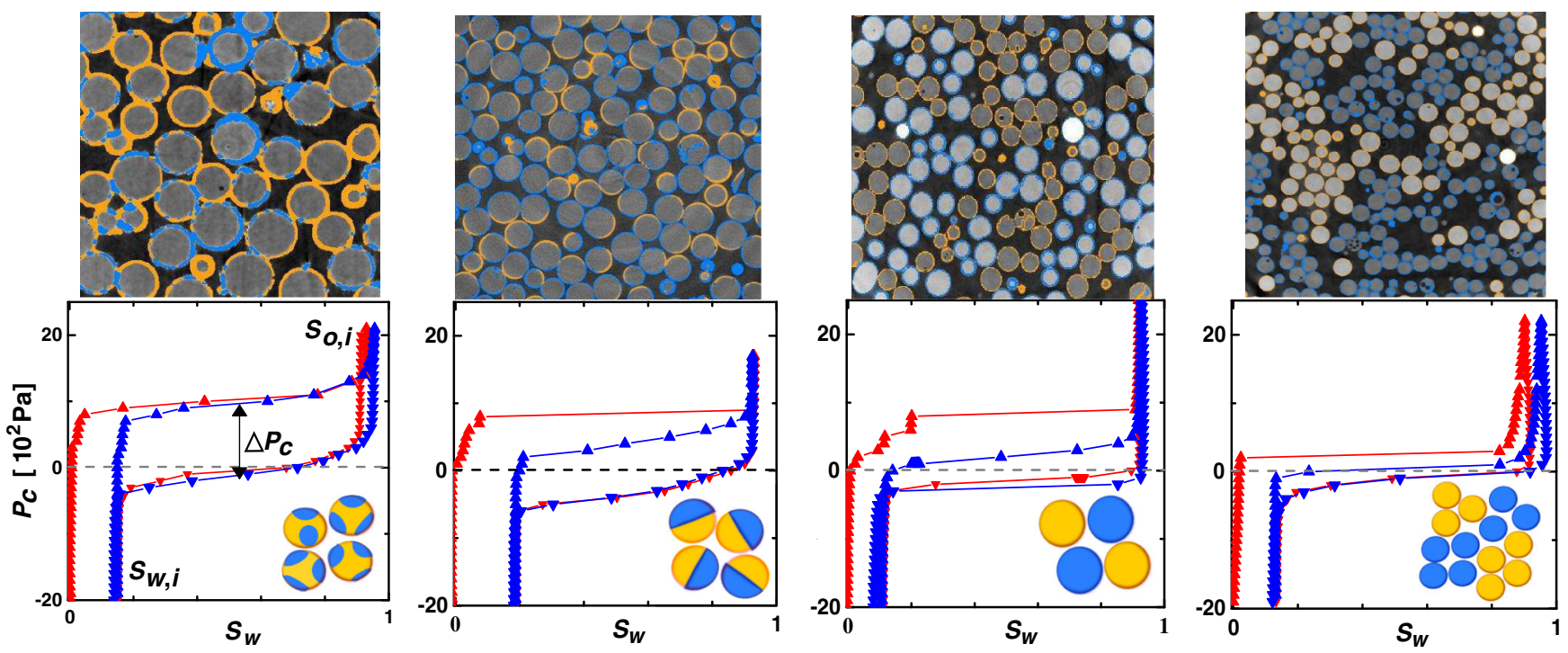

FIG. 5. Representative CPS curves for the patchy, Janus, mixed, and cluster samples. The hysteresis loop opening, $\Delta P_{c}$, as well as the irreducible water $\left(S_{w, i}\right)$ and oil $\left(S_{o, i}\right)$ saturations are indicated on the first graph. X-ray microtomography of dry samples are shown above each curve. Blue and orange are used to represent the two different wettability domains. From these images, the wettability correlation length $\xi$ is calculated. 

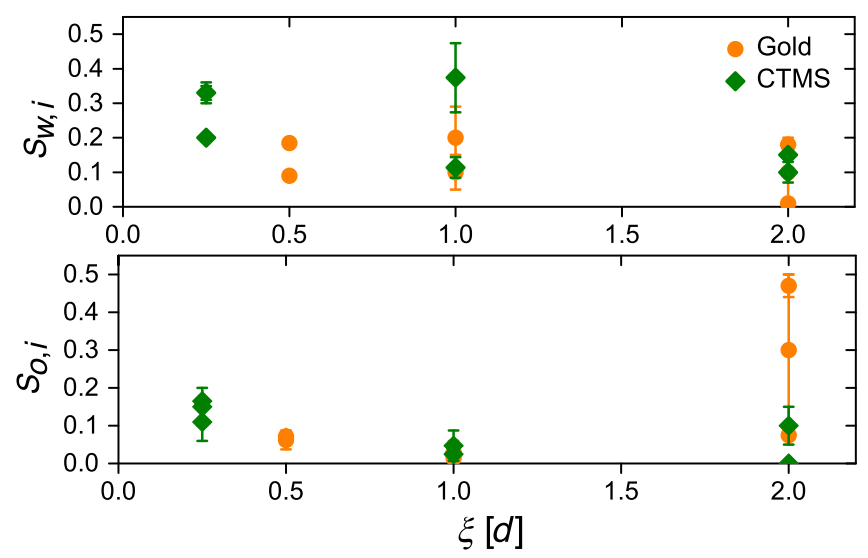

FIG. 6. Irreducible saturations of water (top) and oil (bottom) for samples of different correlation lengths, $\xi$, and different hydrophobic coatings (gold and CTMS). Each data point represents an individual experiment, and the error bars indicate the range of values recorded within a single experiment.

the applied capillary pressure $P_{c}$; see the vertical segments of the CPS curves in Fig. 5. The irreducible water saturation $S_{w, i}$ is the partial volume of water remaining in the packing after a complete oil-invasion branch of the CPS curve. Similarly, the irreducible oil saturation $S_{o, i}$ is the partial volume of oil remaining after a complete water-invasion branch. In our experiments, we do not observe any dependence of the irreducible water and oil saturations on the characteristic length of surface wettability in the bead packs. Figure 6 shows both the water and oil irreducible saturations $S_{w, i}$ and $S_{o, i}$, respectively, plotted against the wetting correlation length $\xi$.

Three-dimensional imaging of oil ganglia trapped in the bead packs using $\mathrm{x}$-ray tomography reveals that large percentages of the residual phase forms a single ganglion whose extension is comparable to the sample size. The volume and morphology of this single large ganglion may vary between different cycles of the experiments explaining observed experimental scatter in $S_{o, i}$ and $S_{w, i}$. Broad volume distributions of the residual phase are typical for slow interfacial displacements and have been reported in other experimental studies [25,27,28,31-33]. Consequently, measurements of irreducible water and oil saturations in small samples display a large scatter.

\section{Hysteresis and dissipation}

In contrast, the typical size of the same-type wetting domains $\xi$ has a direct influence on the hysteresis loop opening $\Delta P_{c}$ defined as the difference between the applied capillary pressure $P_{c}$ on the upper (water invasion) and lower (oil invasion) branch at a water saturation $S_{w}=\left(1-S_{o, i}+S_{w, i}\right) / 2$. The data plotted in Fig. 7 show that the magnitude of $\Delta P_{c}$ monotonously decreases with increasing $\xi$ for both types of coating (gold and CTMS). Experiments with homogeneous water- and oil-wet bead

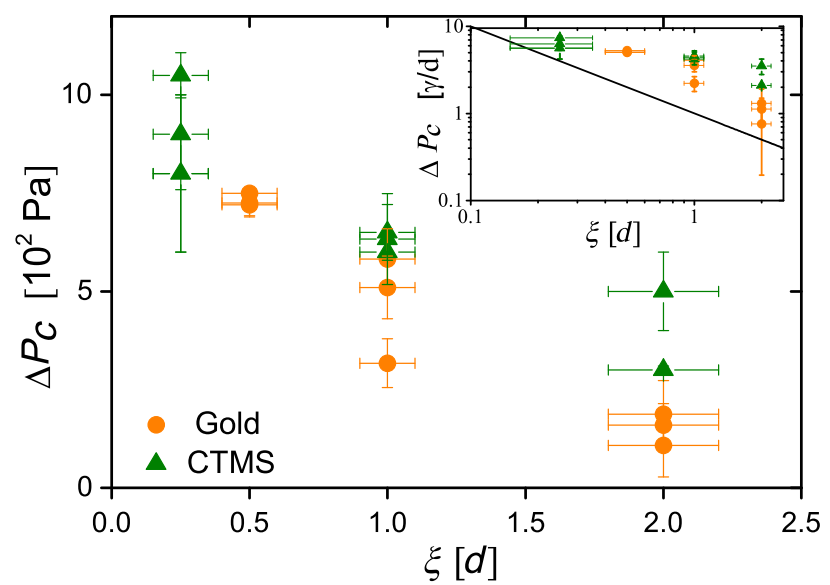

FIG. 7. Hysteresis loop opening $\Delta P_{c}$ for different sample types and coatings against the ratio of wetting domain size $\xi$ to bead diameter $d$. Error bars of $\Delta P_{c}$ indicate variations between successive cycles. The inset shows a rescaling of the same data by the ratio of interfacial tension $\gamma$ and bead diameter $d$. The solid line is given by $\gamma / \xi$.

packs yield values for $\Delta P_{c}$ on the same order as for the mixed and cluster samples $(4 \pm 1) \times 10^{2} \mathrm{~Pa}$. The inset of Fig. 7 shows that the obvious suggestion that $\Delta P_{c}$ might be given by $\gamma / \xi$ (solid line) accounts for the rough trend but does not describe the data appropriately.

The area enclosed by the hysteresis loop, which is approximately $\Delta P_{c}\left(1-S_{o, i}-S_{w, i}\right)$, is a direct measure of the work dissipated during fluid displacement. Capillary hysteresis is a permanent hysteresis; i.e., the dissipation is still present in the limit of a quasistatic interfacial advance. As the width of the loop given by $\left(1-S_{o, i}-S_{w, i}\right)$ is virtually independent on $\xi$, the area of the stable loop decreases when increasing $\xi$. The additional dissipation observed for small $\xi$ can be understood from the pinning and depinning of three-phase contact lines at the boundaries between the water- and oil-wet surface domains. These pinning sites are, if not absent, certainly rare in samples with $\xi>d$, while for samples with $\xi<d$, the density of pinning sites increases as $\xi$ decreases.

Our hypothesis that contact line pinning causes the enhanced capillary hysteresis for $\xi<d$ is further supported by the indication that the topology of the wetting domains affects interfacial displacement. The CPS curve for the patchy sample in Fig. 5 is centered around a higher pressure compared to the other samples. Consistent with all the samples, the surfaces of these beads are $50 \%$ oil and $50 \%$ water wet; however, the hydrophobic part forms a continuous surface, whereas the hydrophilic portion exists as discrete patches. It appears that this topological feature results in an asymmetry of apparent wettability, similar to experimental observations made on planar surfaces with analogous features where contact line pinning at defect sites results in asymmetric contact-angle hysteresis [34]. 


\section{B. Morphology of the liquid front}

In order to differentiate the additional dissipation in the presence of wetting heterogeneities caused by pinning or depinning of the three-phase contact line from other mechanisms, we employ in situ x-ray microtomography imaging of the fluid distributions.

The voxels pertaining to the liquid front are obtained by first segmenting the image into three phases: oil, water, and beads. The volume of segmented water is compared to the fluid saturations, which are known from the mass balance measurements and is in agreement to a few percent. Such external validation is necessary for reducing potential errors arising from shifts in image intensity and user selection of threshold values [35]. A label image of each segmented phase is made, identifying connected components using 3D 26-voxel connectivity criteria (vertex connectivity). The analysis tends to err towards including a droplet which is not actually connected rather than cutting it off from the main liquid body, potentially overestimating the roughness of the interface on a microscopic scale. The continuous water and oil phases, i.e., the liquid volumes that are connected to the membrane being permeable to the respective liquid, are then dilated by 1 voxel (expanded in along all edges by 1 voxel), and the active liquid front is defined as the overlapping voxels between the dilated water and oil regions.

Figure 8 shows the distribution of the aqueous phase (blue) and the active liquid front (red) inside the bead packing (white). We observe a transition to a rougher front shape at approximately $\xi=1$. For samples with $\xi<1$, the front appears to have a more compact shape, whereas in the samples with $\xi>1$, the fronts exhibit a more fingered appearance. This effect can be strongly seen at water saturations of $S_{w} \approx 0.3$ but also persists to higher saturations at $S_{w} \approx 0.5$.

In order to quantitatively characterize the geometry of the active interface, we measure how the volume of the
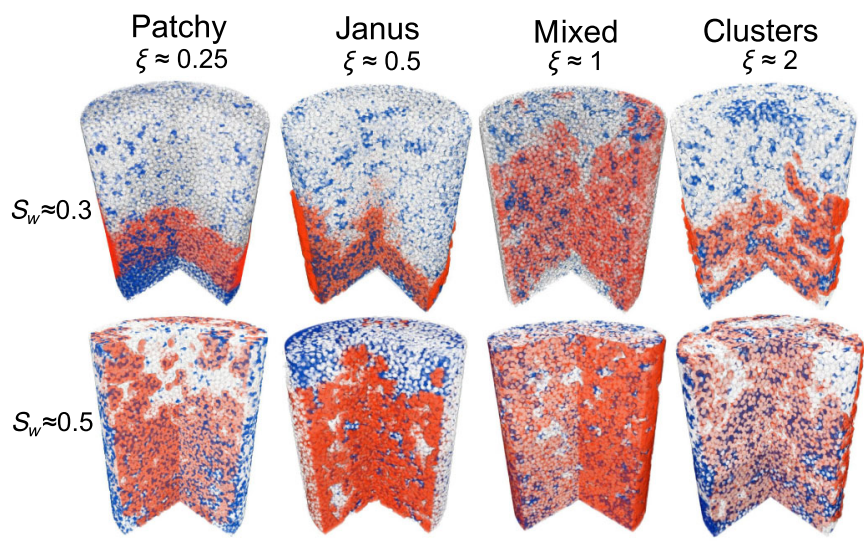

FIG. 8. Aqueous phase (blue) and active front (red) inside the different bead packs imaged during the second aqueous phase invasion, at a saturation of $S_{w} \approx 0.3$ (top) and $S_{w} \approx 0.5$ (bottom). interface (i.e., its first Minkowski measure [36]) grows when we coarse grain the image [37]. For this purpose, we again start from the voxels of the active liquid front. These are the seed points around which spheres of radius $c$ are then placed. Next, the collective volume (the union set of all spheres) of the expanded interface is calculated. Hence, we are counting only once the overlapping regions from neighboring seed voxels. Finally, the volume of the union set of all spheres is normalized by the total volume of the sample.

The growth of the collective volume of the expanded interface with respect to the sphere radius $c$ provides quantitative information on the shape of the liquid front. Seed points, which are dispersed far apart within the packing, for example, due to a fingered interface, will contribute a larger total volume than seed points which are close together. Therefore, the smaller the collective volume of the expanded interface is for a given sphere radius $c$, the smoother is the original interface. In this way, we can quantify the differences between the roughness of liquid fronts in our samples. Figure 9 shows the volume evolution of the active interface while increasing the inflation radius $c$ for the four different samples. Here, we see that our qualitative interpretation from Fig. 8 is confirmed that the active liquid front is smoother in the patchy and Janus samples, and the mixed and cluster samples exhibit a rougher, more fingered active liquid front.

Similar to the CPS curves, we observe a difference in front roughness between the initial imbibition compared with the secondary imbibition or drainage fronts and then again no significant change in front roughness between measurements of the same saturations on successive cycles, as shown in the inset of Fig. 9.

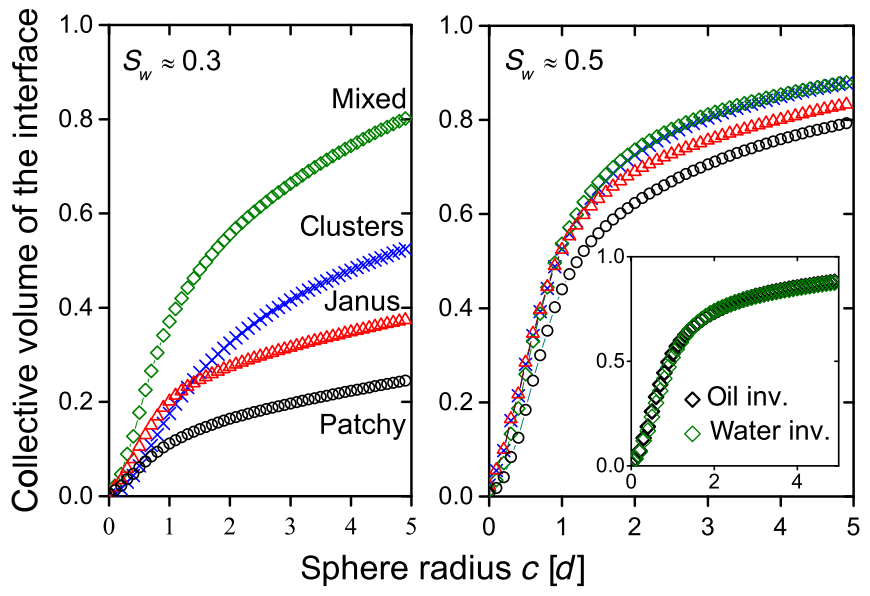

FIG. 9. Quantitative descriptor of the roughness of the active liquid front. The collective volume of the interface is plotted as a function of the coarse-grain sphere diameter c imaged during water invasion with water saturations $S_{w} \approx 0.3$ (left) and $S_{w} \approx 0.5$ (right). The inset shows the curves obtained for water and oil invasion within a mixed packing each at $S_{w}=0.5$. 


\section{Droplet distributions inside bead packs}

An additional dissipation during an interfacial advance in porous media is caused by interfacial pinch-off events. This dissipation mechanism can be also observed in porous media with homogeneously wet walls. In the presence of heterogeneously wettable walls, pinning at the wetting heterogeneities may enhance the formation of small droplets. By comparing the size distribution of disconnected droplets for samples with different correlation lengths, we can test if interfacial pinch-off events contribute to the differences in observed hysteresis.

A statistical analysis of the x-ray tomography images reveals only small qualitative differences in the size distribution of the residual phase between different samples and measurements at different points of CPS curves. Except for very small droplet volumes, the volume distribution of the residual phase shown in Fig. 10(a) follows a power law $n(s) \propto s^{-\tau}$ with an exponent of $\tau=1.3 \pm 0.1$ for volumes $s \lesssim s^{*} \approx 5000$ voxels, while for $s>s^{*}$, the distribution is consistent with the exponent $\tau \approx 2.2$. Both power-law scalings are robust towards image processing. This was rigorously tested by applying morphological filters (erosion, dilation, opening, and closing), which locally change the connectivity of small droplets in order to overestimate potential effects caused by segmentation. The crossover volume $s^{*}$ compares well with a lower estimate of the average pore volume approximately equal to 3000 voxels from a Delaunay tessellation of a random bead packing (radius $R \approx 10$ voxels, packing fraction $\phi \approx 0.6$, average coordination number $N_{c} \approx 6$ ). As residual droplet of volumes $s \gtrsim s^{*}$ extend over several neighboring pores, their formation and frequency can be explained by invasion percolation with trapping predicting $\tau=2.18$ [2]. Similar values of $\tau$ have been recently reported for immiscible fluid displacements in natural sandstones $[25,27,28]$.

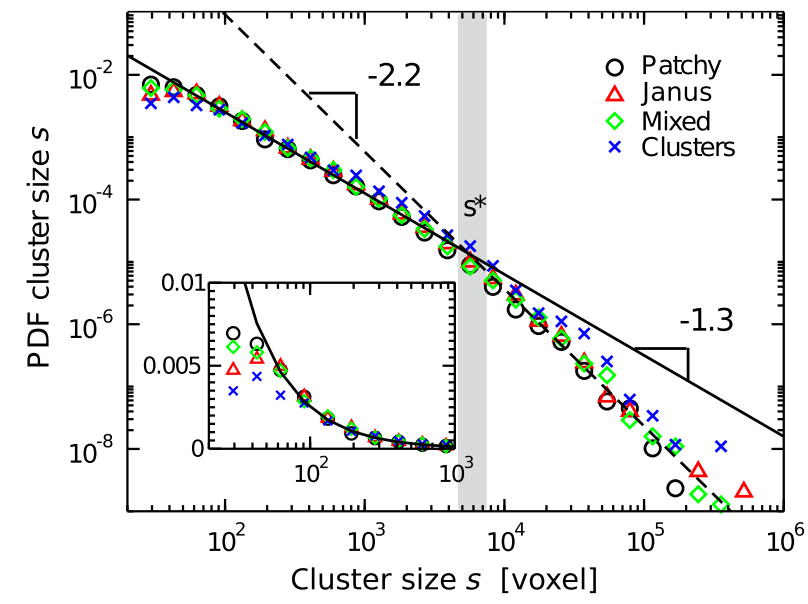

FIG. 10. Frequency $n(s)$ of droplets of volume $s$ for trapped water in partially reinvaded bead packs from x-ray tomography of water invasion, with $S_{w} \approx 0.3$. Data are logarithmically binned to reduce the scatter at large $s$ [38]. Inset: Magnification of $n(s)$ for very small volumes $s$. The voxel size is $17 \mu \mathrm{m}$.
The insensitivity of the volume distributions $n(s)$ with respect to $\xi$ suggests that wetting heterogeneities do not favor an increase of capillary break-up and coalescence events of fluid interface which are a source of dissipation in the displacement processes. Hence, we can conclude that the main contribution to the additional dissipation observed for $\xi<d$ is very likely caused by irreversible pinningdepinning events of the three-phase contact line at boundaries between differently wettable surface domains.

\section{CONCLUSIONS}

In summary, we show that the pore-scale and sub-porescale wettability spatial distribution in a porous media has a profound effect on capillary hysteresis and dissipation during immiscible fluid displacement. By creating a series of heterogeneously wet model porous media from monodisperse bead packs, where only the spatial distribution of wetting heterogeneity is varied, we are able probe the effect of wetting distributions irrespective of global sample wettability and geometric effects.

In both CPS experiments and in situ x-ray tomography imaging, we observe an effect of the typical size of the wetting domains, here quantified by the wetting correlation length $\xi$. The capillary hysteresis loop opening $\left(\Delta P_{c}\right)$ measured by CPS experiments varies monotonically with $\xi$. Larger hysteresis values are measured for $\xi<d$, demonstrating that the dissipation during the water invasion and removal cycle is larger in the presence of multiple small heterogeneities. This result highlights the significance of wetting heterogeneity existing on small length scales. We observe in the tomography images a smoothing of liquid fronts for $\xi<d$. We postulate that these effects are due to pinning of the advancing liquid front at the junctions between wetting surfaces. We do not find a dependence of the irreducible saturations on the wettability distribution. Besides droplets with dimensions well below the pore scale, the volume distribution of residual fluid droplets is barely affected by the length scale of the wetting heterogeneity and still consistent with percolation theory. The independence of droplet distributions on the wettability patterns further supports the idea that local contact line pinning is the underlying mechanism for the enhanced dissipation in bead packs with small $\xi$ and not other irreversible events such as increased pinch-off events.

Future work employing time-resolved $\mathrm{x}$-ray microtomography of interfacial advance and contact line pinning in small ensembles of pores could provide helpful insights into the dissipation mechanisms, emerging droplet distributions, and front morphologies.

\section{ACKNOWLEDGMENTS}

We gratefully acknowledge Thomas Hiller and Daniel Herde for help with the correlation length calculations, Fabian Schaller for help with the Minkowski shape 
descriptors, Mario Scheel and Renaud Dufour for supporting measurements, and scientific discussions and Wolf Keiderling and Udo Krafft for building the experimental cells. We thank Steffen Berg for fruitful discussions regarding the droplet size distribution analysis. We acknowledge generous support from BP Exploration Operation Company Ltd. within the GeoMorph research project.

[1] F. A. L. Dullien, Porous Media: Fluid Transport and Pore Structure (Academic Press, New York, 1991).

[2] M. Sahimi, Flow and Transport in Porous Media and Fractured Rock: From Classical Methods to Modern Approaches (John Wiley \& Sons, New York, 2011).

[3] W. Anderson, Wettability literature survey part 4: Effects of wettability on capillary pressure, J. Pet. Technol. 39, 1283 (1987).

[4] A. J. Kota, G. Kwon, W. Choi, J. M. Mabry, and A. Tueja, Hygro-respondive membranes for efficient oil-water separation, Nat. Commun. 3, 1025 (2012).

[5] M. A. Shannon, P. W. Bohn, M. Elimelech, J. G. Georgiadis, and B.J. Marinas, Science and technology for water purification in the coming decades, Nature (London) 452, 301 (2008).

[6] W. He, J. Yi, and T. Van Nguyen, Two-phase flow model of the cathode of PEM fuel cells using interdigitated flow fields, Am. Instit. Chem. Eng. J. 46, 2053 (2000).

[7] P. K. Sinha, P. P. Mukherjee, and C.-Y. Wang, Impact of GDL structure and wettability on water management in polymer electrolyte fuel cells, J. Mater. Chem. 17, 3089 (2007).

[8] T. M. Squires and S. R. Quake, Microfluidics: Fluid physics at the nanoliter scale, Rev. Mod. Phys. 77, 977 (2005).

[9] N. Champagne, R. Vasseur, A. Montourcy, and D. Bartolo, Traffic jams and intermittent flows in microfluidic networks, Phys. Rev. Lett. 105, 044502 (2010).

[10] C. Cottin, H. Bodiguel, and A. Colin, Influence of wetting conditions on drainage in porous media: A microfluidic study, Phys. Rev. E 84, 026311 (2011).

[11] B. Kozlov, M. H. Schneider, B. Montaron, M. Lagues, and P. Tabeling, Archie's law in microsystems, Transp. Porous Media 95, 1 (2012).

[12] E. Nordgard, G. Sorland, and J. Sjoblom, Behavior of asphaltene model compounds at W/O interfaces, Langmuir 26, 2352 (2010).

[13] T. Hassenkam, L. L. Skovbjerg, and S. L. S. Stipp, Probing the intrinsically oil-wet surfaces of pores in North Sea chalk at subpore resolution, Proc. Natl. Acad. Sci. U.S.A. 106, 6071 (2009).

[14] M. J. Bickle, Geolomedia carbon storage, Nat. Geosci. 2, 815 (2009).

[15] M. T. Balhoff, S. G. Thomas, and M. F. Wheeler, Mortar coupling and upscaling of pore-scale models, Comput. Geosci. 12, 15 (2008).

[16] I. Battiato, D. M. Tartakovsky, A. M. Tartakovsky, and T. Scheibe, Hybrid models of reactive transport in porous and fractured media, Adv. Water Resour. 34, 1140 (2011).
[17] M. Kumar, A. Fogden, T. Senden, and M. Knackstedt, Investigation of pore-scale mixed wettability, SPE J. 17, 20 (2012).

[18] P. Ustohal, F. Stauffer, and T. Dracos, Measurement and modeling of hydraulic characteristics of unsaturated porous media with mixed wettability, J. Contam. Hydrol. 33, 5 (1998).

[19] D. M. O'Carroll, L. M. Abriola, C. A. Polityka, S. A. Bradford, and A.H. Demond, Prediction of two-phase capillary pressure-saturation relationships in fractional wettability systems, J. Contam. Hydrol. 77, 247 (2005).

[20] A. Dixit, J. S. Buckley, S. McDougall, and K. Sorbie, Empirical measures of wettability in porous media and the relationship between them derived from pore-scale modelling, Transp. Porous Media 40, 27 (2000).

[21] I. Fatt and W. Klikoff, Effect of fractional wettability on multiphase flow through porous media, Trans. Am. Inst. Min., Metall. Pet. Eng. 216, 426 (1959).

[22] R. D. Hazlett, S. Chen, and W. Soll, Wettability and rate effects on immiscible displacement: Lattice Boltzmann simulation in microtomographic images of reservoir rocks, J. Pet. Sci. Eng. 20, 167 (1998).

[23] C. Miller, G. Christakos, P. Imhoff, J. McBride, and J. Pedit, Multiphase flow and transport modeling in heterogeneous porous media: Challenges and approaches, Adv. Water Resour. 21, 77 (1998).

[24] S. Berg, H. Ott, S. A. Klapp, A. Schwing, R. Neiteler, N. Brussee, A. Makurat, L. Leu, F. Enzmann, J. O. Schwarz et al., Real-time 3D imaging of Haines jumps in porous media flow, Proc. Natl. Acad. Sci. U.S.A. 110, 3755 (2013).

[25] A. Georgiadis, S. Berg, A. Makurat, G. Maitland, and H. Ott, Pore-scale micro-computer-tomography imaging: Nonwetting-phase cluster-size distribution during drainage and imbibition, Phys. Rev. E 88, 033002 (2013).

[26] S. S. Datta, H. Chiang, T. S. Ramakrishnan, and D. A. Weitz, Spatial fluctuations of fluid velocities in flow through a three-dimensional porous medium, Phys. Rev. Lett. 111, 064501 (2013).

[27] S. Iglauer, S. Favretto, G. Spinelli, G. Schena, and M. J. Blunt, X-ray tomography measurements of power-law cluster size distributions for the nonwetting phase in sandstones, Phys. Rev. E 82, 056315 (2010).

[28] S. Iglauer, M. Ferno, P. Shearing, and M. Blunt, Comparison of residual oil cluster size distribution, morphology and saturation in oil-wet and water-wet sandstone, J. Colloid Interface Sci. 375, 187 (2012).

[29] M. E. Ahmed and P. J. Van Geel, Potential concerns related to using octadecyltrichlorosilane (OTS) in rendering soils and porous ceramics hydrophobic, J. Contam. Hydrol. 110, 22 (2009).

[30] M. Scheel, Ph. D. thesis, Georg August Universität Göttingen, 2009.

[31] M. Prodanovic, W. Lindquist, and R. Seright, Porous structure and fluid partitioning in polyethylene cores from 3D x-ray microtomographic imaging, J. Colloid Interface Sci. 298, 282 (2006).

[32] M. Prodanovic, W. Lindquist, and R. Seright, 3D imagebased characterization of fluid displacement in a Berea core, Adv. Water Resour. 30, 214 (2007). 
[33] S. S. Datta, T. Ramakrishnan, and D. A. Weitz, Mobilization of a trapped non-wetting fluid from a threedimensional porous medium, Phys. Fluids 26, 022002 (2014).

[34] C. Priest, R. Sedev, and J. Ralston, Asymmetric wetting hysteresis on chemical defects, Phys. Rev. Lett. 99, 026103 (2007).

[35] K. A. Culligan, D. Wildenschilde, B. S. Christensen, W. G. Gray, M. L. Rivers, and A. F. Thompson, Interfacial area measurement for unsaturation flow through a porous medium, Water Resour. Res. 40, W12413 (2004).

[36] D. Legland, K. Kieu, and M.-F. Devaux, Computation of Minkowski measures on 2D and 3D binary images, Image Anal. Stereol. 26, 83 (2007).

[37] K. Huang, M. Brinkmann, and S. Herminghaus, Wet granular rafts: Aggregation in two dimensions under shear flow, Soft Matter 8, 11939 (2012).

[38] M. Newman, Power laws, Pareto distribution and Zipf's law, Contemp. Phys. 46, 323 (2005). 